\title{
Perspectives on Anaphylaxis Epidemiology in the United States with New Data and Analyses
}

\author{
Zuber D. Mulla • Robert Y. Lin • Michael R. Simon
}

Published online: 2 November 2010

(C) The Author(s) 2010. This article is published with open access at Springerlink.com

\begin{abstract}
Anaphylaxis incidence rates and time trends in the United States have been reported using different data sources and selection methods. Larger studies using diagnostic coding have inherent limitations in sensitivity and specificity. In contrast, smaller studies using chart reviews, including reports from single institutions, have better case characterization but suffer from reduced external validity due to their restricted nature. Increasing anaphylaxis hospitalization rates since the 1990s have been reported abroad. However, we report no significant overall increase in the United States. There have been several
\end{abstract}

Z. D. Mulla

Department of Obstetrics and Gynecology, Texas Tech University

Health Sciences Center, Paul L. Foster School of Medicine,

El Paso, TX, USA

\section{Z. D. Mulla}

Department of Epidemiology and Biostatistics,

University of South Florida College of Public Health,

Tampa, FL, USA

\section{R. Y. Lin}

Departments of Medicine, New York Downtown Hospital,

New York, NY, USA

R. Y. Lin

New York Medical College,

Valhalla, NY, USA

\section{R. Simon $(\square)$}

Division of Allergy and Clinical Immunology,

Department of Medicine, William Beaumont Hospital,

Royal Oak, MI 48073, USA

e-mail: mrsimonmd@sbcglobal.net

\section{R. Simon}

Departments of Internal Medicine and Pediatrics,

Wayne State University School of Medicine,

Detroit, MI, USA reports of increasing anaphylaxis rates in northern populations in the United States, especially in younger people, lending support to the suggestion that higher anaphylaxis rates occur at higher latitudes. We analyzed anaphylaxis hospitalization rates in comparably sized northern (New York) and southern (Florida) states and found significant time trend differences based on age. This suggests that the relationship of latitude to anaphylaxis incidence is complex.

Keywords Anaphylaxis · Epidemiology · Population based - Latitude - Time trend - ICD-9-CM code - Children . Incidence

\section{Introduction}

Much that has been published under the category of the epidemiology of anaphylaxis has been a description of the syndrome as seen in specific practice venues, such as a hospital or clinic $[1,2]$, rather than the study of anaphylaxis in defined populations. Because of the infrequent occurrence of anaphylaxis and, until recently, the varying definitions of the disorder, no anaphylaxis incidence studies have been prospective cohort studies specific for anaphylaxis. As a result, a great deal remains to be learned about the epidemiology of anaphylaxis.

A substantial portion of the existing data on the epidemiology of anaphylaxis has come from investigations that have limited scope population sources. Examples of these studies include surveys [2,3], the quality of which depends on multiple factors, including the response rate, the sample size, and especially selection bias. Other examples of limited scope inquiries include voluntary registries [4, 5]. Registries are a representative source of epidemiologic data only if the reporting of the condition is mandatory and the data are validated. 
Broad population-based studies, in contrast, pertain to general populations and identify all or a known fraction of the cases in a particular community $[6,7]$. These studies allow descriptive and analytic epidemiologic analyses to be accomplished. Descriptive epidemiology denotes the examination of the frequency of a disease. Analytic epidemiology, on the other hand, is concerned with testing hypotheses and identifying the risk factors and causes of disease [7]. Examples of broad population-based studies include analyses of nationwide/statewide hospital and vital statistics databases [8]. Hospitalization registries use codes such as International Classification of Diseases (ICD)-9 and ICD-10 to identify patients whose principal discharge diagnosis is anaphylaxis. Investigations using these sources of data have more external validity (ie, generalizability) than limited scope studies. Anaphylaxis hospitalization studies have involved primarily large administrative hospitalization databases. These studies have been especially useful in characterizing time trends, as clinical practice and principal discharge diagnosis coding are unlikely to have changed significantly during the study periods involved.

A weakness of such studies is that there is little or no verification of the diagnosis. Therefore, there are attendant risks of overdiagnosis and, especially, underrecognition. This problem with internal validity is counterbalanced by a greater generalizability due to the data coming from a broad range of locations and other demographic characteristics. Nonetheless, allergy researchers should strive to conduct studies that have internal and external validity. Investigations that are free of bias (ie, systematic error) have internal validity, whereas those whose results are generalizable have external validity. These nationwide/statewide hospital discharge databases probably are indicators of more severe anaphylaxis. They do not include patients treated for anaphylaxis as outpatients or as "treat-and-release" emergency department (ED) patients. Anaphylaxis patients seen in hospitals, EDs, health maintenance organizations, and physicians' practices also have been identified using anaphylaxis ICD-9 coding as well as diagnostic algorithms.

Chart review to identify cases has been used for case verification and to estimate the sensitivity and specificity of code-based case findings. Chart review studies can detail organ/system involvement for anaphylaxis and allow for identification of more cases, many of which are not encountered in hospitalization or ED records. Thus, these studies probably better characterize the overall incidence of anaphylaxis. Chart reviews of community data indicate that at least $39 \%$ of anaphylaxis cases are not treated as inpatients [9]. The previous lack of consensus about the definition/grading of anaphylaxis has added to the difficulty in assessing and comparing studies and their case identification methods [10].

\section{Population-based Studies of Anaphylaxis}

There are relatively few population-based studies of anaphylaxis in the United States. The first such study described anaphylaxis in the ED in the tertiary referral hospital in Rochester, MN, which has a population of about 71,000 people [11]. ED visits were studied over a 4-month period in 1993, and an incidence of 90 per 100,000 per year based on presenting signs and symptoms was reported [11, 12]. A follow-up study by the same investigators encompassed a 5-year period (1983-1987) [12]. A total of 1255 medical records were reviewed for diagnoses suggestive of anaphylaxis. These diagnoses included food allergy, drug allergy, hypersensitivity not otherwise specified, adverse effect of immunotherapy, adverse effect of injectable diagnostic agent, subglottic or pharyngeal edema, bee sting allergy, and unspecified allergic reaction. However, during this 5 -year period, only 154 anaphylactic episodes among 133 individual patients were identified. The average annual incidence was 21 per 100,000 per year. A partial explanation for the very high incidence reported in the 1993 study may be that the data were obtained during the months of May through August (Harduar-Morano, L., personal communication). The incidence of anaphylaxis in Olmsted County, MN, from 1983 through 1987 was seasonally increased to a mean of 21 per month during July and August, as compared with an incidence as low as 3 in April. Sixty-five of 133 cases (49\%) occurred during May through August [12]. If the incidence were to be adjusted for the seasonal skewing of data, the revised ED incidence would then be about two thirds as much (ie, 60 per 100,000). This ED incidence is similar to that reported for 2000 for all provider visits in a later Olmsted County publication [13]. The major strength of these first two studies is that this population is served by a single medical system (the Mayo Clinic), which allows for medical record review. Weaknesses include the relatively small population and the limited demographic range of that population. Unfortunately, a weakness of the third of the Mayo Clinic studies is that the case finding methods are unclear.

Population-based studies of larger populations have been accomplished in the United Kingdom [14, 15]. This has been facilitated by the National Health System, which records disease information for the entire country. The incidence of anaphylaxis in the United Kingdom has been reported to be 8.4 per 100,000 in 1994 to 1999 [14] and 7.9 per 100,000 in 2005 [15]. This compares with the US incidence in an HMO population of 19.1 per 100,000 (1996-2006) [16•]. The strength of these reports is the large population studied. The weakness is that these studies depended on the lack of an accepted case definition and diagnoses made by physicians who are not experts in anaphylaxis. Weaknesses, such as lack of expert diagnosis 
and deficiencies in coding, affect all studies of anaphylaxis to varying degrees.

Three population-based studies of anaphylaxis in Florida have been conducted $[8,17 \bullet, 18 \cdot]$. ED visits have been assessed using both ICD-9-CM (Clinical Modification) coding for an anaphylaxis diagnosis and an algorithm that derives an anaphylaxis diagnosis on the basis of signs and symptoms in patients for whom the treating physician did not arrive at the diagnosis [18•]. This study found an incidence of ED visits of 3.3 per 100,000 persons per year when anaphylaxis ICD-9-CM coding was used. An additional 4.4 per 100,000 persons per year were identified using the algorithm. Additional studies of essentially the same Florida population found 2.8 hospital discharges for anaphylaxis per 100,000 population per year [8] and 5 anaphylactic deaths per 10 million population per year [17॰].

This latter study determined the 10-year incidence of death from anaphylaxis in Florida. Florida residents who died from anaphylaxis from 1996 and 2005 were identified from ICD-9 and ICD-10 codes on death certificates statewide. Age, race, and gender-specific anaphylactic death rates were calculated. There were 89 deaths. Decedents with autopsy-confirmed diagnoses did not differ from those with clinical diagnoses with regard to race, anaphylactic triggers, or the presence of heart or lung disease. The relative risk of death from anaphylaxis was 14.09 for individuals 65 years of age and older $(P=0.0000002)$ and 6.10 for people 35-64 years of age $(P=0.001)$ compared with those who were $5-14$ years of age. Deaths that occurred in EDs or outpatient settings were 2.11 times as likely to be anaphylactic deaths than deaths that occurred in inpatient settings $(P=0.0026)$. The ratios of anaphylactic deaths to total deaths in March and April and in July and August were greater than the ratios for the other bimonthly periods $(P=0.02)$.

The Mayo clinic studies-from a smaller population base-reported one death from anaphylaxis during the 5year period from 1983 to 1987 [12] and no deaths during the 10-year period from 1990 to 2000 [13]. These results are essentially equivalent and not inconsistent with the Florida finding of 0.05 deaths per 100,000 per year [17•].

The British reports indicate 1.25-3.73 anaphylactic deaths per 10 million per year $[14,19]$, which is consistent with both the Florida and Minnesota mortality rates that are based on ICD-9-CM coding for case finding. The twofold higher rate of 11 per 10 million in Bern County, Switzerland, may reflect fewer missed diagnoses [20].

\section{Is the Prevalence of Anaphylaxis Increasing?}

Increases in the anaphylaxis hospitalization rate have been reported in the United Kingdom, United States, and Australia. In Australia, the increased anaphylaxis hospital- ization incidence was driven by hospitalizations in young children $(<5$ years of age), in whom food anaphylaxis was the most common subset of anaphylaxis [21]. The hospitalization rate in young children increased from 15 per 100,000 in 1993-1994 to 25 per 100,000 in 2004-2005 $(8.8 \% / y)$, while the overall age-adjusted hospitalization rate increased from less than 4 per 100,000 to 10 per 100,000 .

In the United Kingdom, admission rates for anaphylaxis rose sevenfold from 0.5 per 100,000 population in 1990 1991 to 3.6 in 2003-2004, and increases were noted for all age groups [22]. These data highlight large differences in hospitalization rates in the different countries that may be the result of different admission criteria as well as age subset differences.

For this report, we determined the secular trend in anaphylaxis discharges in the United States. Lin and Shah [23] reported total hospitalizations for a principal diagnosis of anaphylaxis due to food (ICD-9-CM 995.6), nonfood anaphylaxis (ICD-9-CM 995.0), and anaphylaxis due to serum (ICD-9-CM 999.4) for the years 1998 through 2005. We calculated a population-based rate for the entire United States using these data together with the respective yearly US population estimates (Table 1). Year was treated as a continuous exposure variable (independent variable) in a negative binomial regression model. The relative rate (RR) for the relationship between year and anaphylaxis hospitalizations was 1.01 , indicating a $1 \%$ increase in the rate for each 1-year increase with anaphylaxis rates per 100,000, rising from 2.1 to 2.3 ( $P=0.24$ for the time trend).

There have been two more geographically limited US reports of a secular increase in anaphylaxis. In one, the annual incidence of anaphylaxis in Rochester, MN, is reported to have increased modestly from slightly less than 50 per 100,000 in 1990 to 60 per 100,000 in $2000(P=0.034)$ [13]. However, because of lack of clarity regarding the case finding methods and the large year-to-year fluctuations in anaphylaxis incidence reported, it is difficult to draw conclusions regarding a secular trend from these data. Interestingly, there was no increase in anaphylaxis from 1983 through 1987 in Olmsted County, MN [12]. The other geographically limited study showed an increase in hospitalizations in children in New York State from 1990 through 2006 [24]. Hospitalizations for anaphylaxis in adults were not reported.

Because of these concerns, we decided to further test the hypothesis that there has been a secular increase in anaphylaxis in the United States. The New York State findings in children were further assessed by adjusting the case definition and repeating the analysis using New York State and Florida databases [24]. New York and Florida have similarly sized populations (15-20 million people). Our new analysis was performed using negative binomial regression models. Time trends were assessed by treating 
Table 1 Number of hospitalizations due to anaphylaxis and annual rate in the United States (1998-2005) ${ }^{\mathrm{a}}$

\begin{tabular}{lccc}
\hline Year & Hospitalizations, $n$ & US population, $n$ & Rate per 100,000 population \\
\hline 1998 & 5583 & $270,248,000$ & 2.07 \\
1999 & 5863 & $272,691,000$ & 2.15 \\
2000 & 5938 & $282,171,957$ & 2.10 \\
2001 & 5681 & $285,081,556$ & 1.99 \\
2002 & 5907 & $287,803,914$ & 2.05 \\
2003 & 5807 & $290,326,418$ & 2.00 \\
2004 & 6365 & $293,045,739$ & 2.17 \\
2005 & 6670 & $295,753,151$ & 2.26 \\
\hline
\end{tabular}

${ }^{\text {a }}$ Principal diagnosis of anaphylaxis due to food (code 995.6*), nonfood anaphylaxis (code 995.0), or anaphylaxis due to serum (code 999.4) from Lin and Shah [23]. Relative rate was 1.01 ( $P$ for the $1 \%$ increase in the anaphylaxis hospitalization rate for each increase of 1 calendar year, 0.24 ); from a negative binomial regression model treating year as continuous independent variable

the calendar year as a continuous variable. Principal and secondary discharge diagnosis fields were searched for the following ICD-9-CM codes denoting anaphylaxis: 995.0, 995.6, and 999.4. The denominators for the anaphylaxis hospitalization rates were the state resident population estimates [25-27]. These analyses confirmed a statistically significant increase in anaphylaxis hospitalizations for individuals younger than 20 years of age from 1996 to 2005 in New York State (Fig. 1). That increase is from 2.1 to 3.7 per 100,000 per year. For each 1-year increase in year, there was a $7 \%$ increase in the anaphylaxis hospitalization rate $(P<0.0001)$ according to a negative binomial regression model. However, between 1996 and 2005, there were no statistically significant changes in the annual anaphylaxis hospitalization rates among Florida children $(P=0.90)$. We also assessed hospitalizations for adults in
New York State and Florida for the same time periods. There was a statistically significant increase in hospitalizations for adults (> 19 years of age) from 1996 to 2005 in New York. That increase is from 2.2 to 2.8 per 100,000 per year. For each 1 -year increase in year, there was a $2 \%$ increase in the anaphylaxis hospitalization rate $(P=0.04)$ according to a negative binomial regression model. The change in rates over time was different for adults and children in New York State, as evidenced by a significant interaction term that was created by multiplying the age variable (children vs adults) by the year of discharge variable $(P=0.0008)$. In contrast to these results, there was no increase in hospitalizations for anaphylaxis in Florida from 1996 through 2005 for children (rates in both years, 1.3 per 100,000 per year; $P=0.90$ ) or adults (rates in both years, 3.1 per 100,000 per year; $P=0.10$ ) (Fig. 1). In

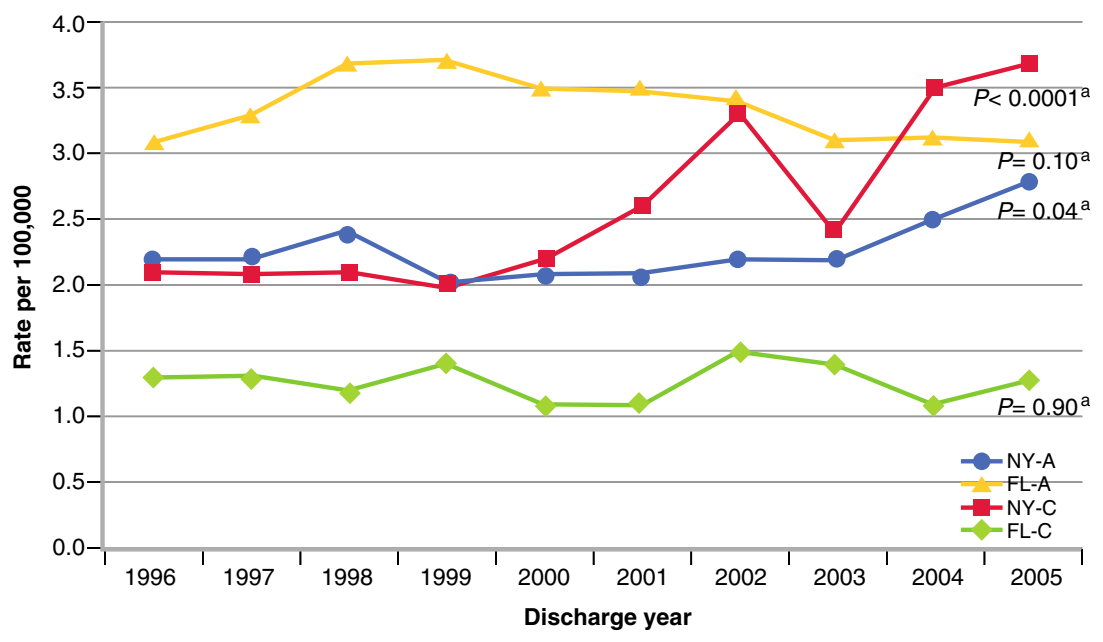

Fig. 1 Anaphylaxis hospitalization rates for residents of New York and Florida between 1996 and 2005. Rates among New York children and adolescents (NY-C) and New York adults (NY-A) are reported along with rates among Florida children and adolescents (FL-C) and Florida adults (FL-A). Children and adolescents were 0 to 19 years of age, and adults were 20 years of age and older. Time trends were evaluated by entering the discharge year as a continuous variable in negative binomial regression models. ${ }^{\text {a }}$ The $P$ values are for change in rate for each 1-year increase in discharge year from a negative binomial regression model 
summary, significant time trends were detected in New York State, but not in Florida.

An attractive explanation for the discrepancy between the studies reporting an increasing incidence in anaphylaxis and our finding that there is no such increase is that the reports showing an increase came from high latitude states (New York and Minnesota). As we reported above, there was no increase in Florida, which is a lower latitude state. It is possible that the lack of a significant increase in anaphylaxis hospitalizations nationally is due to the increase in anaphylaxis hospitalizations in high latitude states being offset by the lack of increase in lower latitude states. This hypothesis, however, requires further testing.

\section{Influence of Latitude on Regional Differences}

Regional differences in anaphylaxis-related clinical encounter and epinephrine prescription prevalence rates have pointed toward an interesting north-south dichotomy. This has led certain investigators to hypothesize a relationship between vitamin D levels and anaphylaxis rates. In a 2004 US study, epinephrine autoinjector prescriptions were used as a surrogate marker for anaphylaxis prevalence [28]. It was found that 8-12 epinephrine autoinjector prescriptions were issued per 1000 population in the New England states, compared with only 2-3 per 1000 in many contiguous southern and southwestern states (from California to Mississippi). Multivariate adjustments for potentially confounding factors did not abrogate the significant difference in epinephrine autoinjector prescriptions noted between the New England states and the rest of the United States (adjusted ORs, 3.55-4.07).
Some of these investigators then examined epinephrine autoinjector prescriptions in 10 major areas in Australia between 2006 and 2007 [29]. They found that stronger geographic differences were noted for children 14 years of age or younger, and an inverse relationship existed between latitude (which are negative numbers below the equator) and the number of epinephrine autoinjectors per 100,000 population. Multivariate adjustments failed to abrogate this relationship. Australian hospitalizations for anaphylaxis between 2002 and 2007 did not, however, show a statistically significant correlation with latitude.

ED visits for acute allergic reactions (a portion of which were anaphylaxis) in the United States between 1993 and 2005 were also examined by this same team [30]. Compared with the southern states, the Northeast had a modest increase in acute allergic reactions (OR, 1.13; 95\% CI, 1.01-1.27), which was only apparent in a multivariate modeling (using demographic factors only). Somewhat greater differences were noted if only food-related allergic reactions were analyzed (OR, 1.33; 95\% CI, 1.14-1.56).

For this report, we decided also to examine regional differences in anaphylaxis hospitalizations using Florida and New York State inpatient discharge databases. We examined regional and time trends in anaphylaxis hospitalizations. Geographical and temporal associations were quantified using RRs calculated from negative binomial regression models that incorporated offset variables [31, 32]. Among individuals $0-19$ years of age, New York State residents were twice as likely as Florida residents to be hospitalized for anaphylaxis (unadjusted RR, 2.07; $P<0.0001$ ) (Table 2). However, after adjusting for the year of discharge and a state-of-residence-by-year-of-discharge interaction term, the RR decreased to $1.38(P=0.01)$. This association reversed direction in individuals 20 years of age and older, with

Table 2 Unadjusted and adjusted relative rates for anaphylaxis hospitalization during the period 1996 to 2005 comparing New York State residents with Florida residents

\begin{tabular}{|c|c|c|c|c|c|c|}
\hline \multirow[t]{3}{*}{ Variables(s) adjusted for: } & \multicolumn{6}{|l|}{ Age of residents } \\
\hline & \multicolumn{3}{|l|}{$0-19$ y } & \multicolumn{3}{|l|}{$\geq 20 \mathrm{y}$} \\
\hline & $\mathrm{RR}\left(\mathrm{NY}\right.$ vs FL) ${ }^{\mathrm{a}}$ & $95 \% \mathrm{CI}$ & $P$ value & $\mathrm{RR}\left(\mathrm{NY}\right.$ vs FL) ${ }^{\mathrm{a}}$ & $95 \% \mathrm{CI}$ & $P$ value \\
\hline (None) & 2.07 & $1.74-2.47$ & $<0.0001$ & 0.67 & $0.63-0.73$ & $<0.0001$ \\
\hline Year of discharge $\mathrm{e}^{\mathrm{b}}$ & 2.06 & $1.80-2.36$ & $<0.0001$ & 0.67 & $0.63-0.73$ & $<0.0001$ \\
\hline Year of discharge, and state-by-year interaction ${ }^{\mathrm{c}}$ & 1.38 & $1.07-1.79$ & 0.01 & 0.57 & $0.49-0.65$ & $<0.0001$ \\
\hline
\end{tabular}

${ }^{a}$ RRs are from negative binomial regression models and stratified by patient age ( $0-19$ and $\geq 20$ years). If adjustment for 1 or more variables resulted in a state (NY vs FL) RR that deviated by $\geq 10 \%$ from the unadjusted state RR, then confounding was considered to be present and the adjusted RR was emphasized

${ }^{\mathrm{b}}$ Year was treated as a continuous variable, with 1 representing 1996, 2 representing 1997, and so forth

${ }^{\mathrm{c}}$ A product interaction term was created by multiplying the state term (NY vs FL) by the year of discharge term. The interaction terms for the $0-19$ and $\geq 20$ age groups were statistically significant $(P=0.001$ and $P=0.01$, respectively)

FL—Florida residents; NY—New York State residents; RR — relative rate 
older New Yorkers experiencing a 43\% lower rate of anaphylaxis hospitalization than older Floridians (adjusted RR, 0.57; $P<0.0001$ ) (Table 2). Adults 20 years of age or older were significantly more likely than children 0-19 years of age to be hospitalized for anaphylaxis in Florida (unadjusted RR, 2.67) (Table 3). The adjusted RR for Florida adults versus Florida children did not deviate by $10 \%$ or more from the unadjusted RR after adjusting for the year of discharge, or adjusting for both the year of discharge and an interaction term [33]. Therefore, confounding by these variables was considered to be absent, and the unadjusted RR of 2.67 was the statistic of interest. In New York State, adults 20 years of age or older were at a lower risk of anaphylaxis hospitalization compared with children 0-19 years of age after adjusting for the year of discharge (adjusted RR, 0.88; $P=0.02$ ), but not after adjusting for both the year of discharge and the age-by-year interaction (adjusted RR, 1.18; $P=0.10$ ) (Table 3 ).

\section{Accuracy of the ICD-9-CM Coding of Anaphylaxis}

The ICD-9-CM codes that include the word anaphylactic are 995.0 (other anaphylactic shock not otherwise specified), 995.6 (anaphylactic shock due to adverse food reaction), and 999.4 (anaphylactic shock due to serum not otherwise specified). The ICD-10 codes that correspond to these are T78.2, T78.0, and T80.5, respectively. There is an additional ICD-10 code for anaphylactic shock due to drugs, T88.6. ICD-9 codes that are commonly used to identify anaphylaxis include shock due to anesthesia (995.4) [9], dermatitis due to food taken internally (693.1), toxic effect of venom (989.5), venomous bite or sting (E905.9), and toxic effect of noxious substances eaten as food (988.*). Use of additional anaphylaxis nonspecific codes has enhanced sensitivity in case findings but at a cost of decreased specificity.

Hospital discharge databases have served as a source of health data for epidemiologic studies for many years [34]. The accuracy of the coding of both discharge diagnoses and procedures in these databases has been debated and evaluated by multiple investigators [34-38].

Two related concepts merit definition at this point: accuracy and reliability. Accuracy is a measure of validity, whereas reliability is a measure of reproducibility [39]. Reliability may be present even though validity is not. Validation of the ICD-9-CM coding of anaphylaxis in hospital inpatient and ED discharge databases would ideally proceed as it has for other serious conditions [34, 37, 38]. First, a large representative sample of records would be extracted. Second, an accepted case definition would be identified as the gold standard. Finally, data would be arranged in a contingency table, and the sensitivity and specificity of the ICD-9-CM coding of anaphylaxis as a principal or secondary discharge diagnosis would be calculated using the accepted case definition.

Klein and Yocum [11] conducted a retrospective analysis of the ED records of all the patients who presented to the ED of a rural tertiary care medical center during a 4-month period. They identified 17 cases of anaphylaxis. However, only four of the 17 patients had a diagnosis of anaphylaxis. The authors concluded, “... most of the cases in this study would have been unreported in the International Classification of Diseases-ninth revision nomenclature as anaphylaxis."

Algorithms incorporating various ICD-9-CM codes have been developed using South Carolina and Florida data to better identify cases of anaphylaxis found in statewide ED databases [18•, 40]. The South Carolina study focused on drug-related anaphylaxis in children and adolescents whose

Table 3 Unadjusted and adjusted relative rates for anaphylaxis hospitalization during the period 1996 to 2005 comparing adults 20 years of age or older with children/adolescents 0 to 19 years of age

\begin{tabular}{|c|c|c|c|c|c|c|}
\hline \multirow[t]{2}{*}{ Variables(s) adjusted for: } & \multicolumn{3}{|l|}{ Florida residents } & \multicolumn{3}{|l|}{ New York State residents } \\
\hline & $\mathrm{RR}$ (adults vs children) ${ }^{\mathrm{a}}$ & $95 \% \mathrm{CI}$ & $P$ value & RR (adults vs children) ${ }^{\mathrm{a}}$ & $95 \% \mathrm{CI}$ & $P$ value \\
\hline (None) & 2.67 & $2.41-2.95$ & $<0.0001$ & 0.87 & $0.75-1.01$ & 0.06 \\
\hline Year of discharge $^{\mathrm{b}}$ & 2.67 & $2.42-2.94$ & $<0.0001$ & 0.88 & $0.79-0.98$ & 0.02 \\
\hline $\begin{array}{l}\text { Year of discharge, and age-by-year } \\
\text { interaction }^{c}\end{array}$ & 2.87 & $2.32-3.55$ & $<0.0001$ & 1.18 & $0.97-1.43$ & 0.10 \\
\hline
\end{tabular}

\footnotetext{
${ }^{a}$ RRs are from negative binomial regression models and stratified by state of residence. If adjustment for 1 or more variables resulted in an RR (adults vs children) that deviated by $\geq 10 \%$ from the unadjusted RR, then confounding was considered to be present and the adjusted RR was emphasized

${ }^{\mathrm{b}}$ Year was treated as a continuous variable in which 1 represented 1996, 2 represented 1997, and so forth

${ }^{\mathrm{c}}$ A product interaction term was created by multiplying the dichotomous age term ( $\geq 20 \mathrm{y}$ vs $\left.0-19 \mathrm{y}\right)$ by the year of discharge term. The interaction term in the Florida model was not statistically significant $(P=0.45)$, but it was significant in the New York model $(P=0.001)$

$\mathrm{RR}$-relative rate
} 
encounters were captured by the South Carolina Emergency Room Hospital Discharge Database during the years 20002002 [40]. The authors' algorithm had a sensitivity of $100 \%$ (95\% CI, 79\%-100\%) and a specificity of $28 \%(95 \% \mathrm{CI}$, $16 \%-43 \%$ ) when compared with their gold standard, which was record review by two physicians.

The Florida team of researchers accessed statewide ED data for the years 2005 and 2006 from the Florida Agency for Health Care Administration [18•]. Patients with anaphylaxis were identified using two methods: ICD-9-CM codes specifically indicating anaphylaxis and an ICD-9-CM algorithm based on the definition of anaphylaxis proposed at the 2005 National Institute of Allergy and Infectious Diseases and the Food Allergy and Anaphylaxis Network symposium [10]. The algorithm identified anaphylaxis if respiratory compromise or skin/mucosal involvement was found in conjunction with shock due to anesthesia, if respiratory compromise or reduced blood pressure was found in conjunction with skin/mucosal involvement, or if a combination of gastrointestinal symptoms and/or respiratory compromise and/or reduced blood pressure and/or skin/ mucosal involvement occurred in association with a potential allergenic exposure. A total of 1149 patients were identified using anaphylaxis ICD-9-CM codes, while 1602 patients were identified with the algorithm. A total of 26 patients were identified by both methods and were included in the ICD-9-CM code total. The authors noted that the clinical risk factors and the demographic features of the cases were consistent between the two methods, although the algorithm was more likely to identify older individuals $(P<0.0001)$, those with hypertension or heart disease $(P<0.0001)$, and individuals with venom-induced anaphylaxis $(P<0.0001)$.

\section{Conclusions}

Population-based studies provide information on diseases in defined populations. Accurate information regarding disease incidence and description can be obtained if these populations are large and diverse. Population-based studies of anaphylaxis in the United States include those in Rochester, MN [11-13]; New York State [24]; and Florida [8, 17•, 18•]. We have added new population-based analyses in this report. There have been reports that the incidence of anaphylaxis has manifested a secular increase in Australia, the United Kingdom, and the United States [13, 21, 22]. We further investigated this phenomenon and were able to confirm an increase in anaphylaxis hospital discharges in New York State, but not in Florida. This suggests an effect of latitude on the increase in anaphylaxis. It had been previously suggested that anaphylaxis is more prevalent in higher latitudes. We found that children in New York State had a higher incidence of hospital discharges for anaphylaxis than children in Florida. However, the anaphylaxis hospital discharge incidence for older individuals was greater in Florida than in New York State. This indicates that the relationship of latitude to the incidence of anaphylaxis is more complex than initially proposed [28].

Disclosure No potential conflicts of interest relevant to this article were reported.

Open Access This article is distributed under the terms of the Creative Commons Attribution Noncommercial License which permits any noncommercial use, distribution, and reproduction in any medium, provided the original author(s) and source are credited.

\section{References}

Papers of particular interest, published recently, have been highlighted as:

- Of importance

1. An epidemiologic study of severe anaphylactic and anaphylactoid reactions among hospital patients: methods and overall risks. The International Collaborative Study of Severe Anaphylaxis. Epidemiology 1998, 9:141-146.

2. Mullins RJ: Anaphylaxis: risk factors for recurrence. Clin Exp Allergy 2003, 33:1033-1040.

3. Laxenaire MC, Mertes PM; Groupe d'Etudes des Réactions Anaphylactoïdes Peranesthésiques: Anaphylaxis during anaesthesia. Results of a two-year survey in France. Br J Anaesth 2001, 87:549-558.

4. Bock SA, Muñoz-Furlong A, Sampson HA: Fatalities due to anaphylactic reactions to foods. J Allergy Clin Immunol 2001, 107:191-193.

5. Moneret-Vautrin DA, Kanny G, Parisot L: First survey from the "Allergy Vigilance Network": life-threatening food allergies in France. Allerg Immunol (Paris) 2002, 34:194-198.

6. A Dictionary of Epidemiology, edn 5. Edited by Porta M. New York: Oxford University Press; 2008:187.

7. Hennekens $\mathrm{CH}$, Buring JE, eds: Epidemiology in Medicine. Boston, MA: Little, Brown and Co.; 1987:81.

8. Mulla ZD, Simon MR: Hospitalizations for anaphylaxis in Florida: epidemiologic analysis of a population-based dataset. Int Arch Allergy Immunol 2007, 144:128-136.

9. Bohlke K, Davis RL, DeStefano F, et al.; Vaccine Safety Datalink Team: Epidemiology of anaphylaxis among children and adolescents enrolled in a health maintenance organization. J Allergy Clin Immunol 2004, 113:536-542.

10. Sampson HA, Muñoz-Furlong A, Campbell RL, et al.: Second symposium on the definition and management of anaphylaxis: summary report-Second National Institute of Allergy and Infectious Diseases/Food Allergy and Anaphylaxis Network symposium. J Allergy Clin Immunol 2006, 117:391-397.

11. Klein JS, Yocum MW: Underreporting of anaphylaxis in a community emergency room. J Allergy Clin Immunol 1995, 95:637-638.

12. Yocum MW, Butterfield JH, Klein JS, et al.: Epidemiology of anaphylaxis in Olmsted County: a population-based study. J Allergy Clin Immunol 1999, 104:452-456.

13. Decker WW, Campbell RL, Manivannan V, et al.: The etiology and incidence of anaphylaxis in Rochester, Minnesota: a report from the Rochester Epidemiology Project. J Allergy Clin Immunol 2008, 122:1161-1165. 
14. Peng MM, Jick H: A population-based study of the incidence, cause, and severity of anaphylaxis in the United Kingdom. Arch Intern Med 2004, 164:317-319.

15. Sheikh A, Hippisley-Cox J, Newton J, Fenty J: Trends in national incidence, lifetime prevalence and adrenaline prescribing for anaphylaxis in England. J R Soc Med 2008, 101:139-143.

16. - Iribarren C, Tolstykh IV, Miller MK, Eisner MD: Asthma and the prospective risk of anaphylactic shock and other allergy diagnoses in a large integrated health care delivery system. Ann Allergy Asthma Immunol 2010, 104:371-377. This was a highquality cohort study of the incidence of anaphylaxis in a regional US population. While its objective was to determine the incidence of anaphylaxis in an asthma cohort, it also provides the incidence of anaphylaxis in the nonasthmatic referent cohort of more than 500,000 people.

17. - Simon MR, Mulla ZD: A population-based epidemiologic analysis of deaths from anaphylaxis in Florida. Allergy 2008, 63:1077-1083. This is the only US population-based study of anaphylactic deaths in a large population (>15 million people). It is the only study to determine the relative risk of death from anaphylaxis by age and location. It also documents that anaphylactic deaths occur preferentially during certain months.

18. - Harduar-Morano L, Simon MR, Watkins S, Blackmore C: Algorithm for the diagnosis of anaphylaxis and its validation using population-based data on emergency department visits for anaphylaxis in Florida. J Allergy Clin Immunol 2010, 126:98104.e4. This study presents and validates an ICD-9-CM-based diagnostic algorithm that allows for a more accurate estimate of anaphylaxis incidence by capturing previously unidentified cases, especially venom-induced cases. The use of the algorithm more than doubles the number of cases identified.

19. Pumphrey RS: Fatal anaphylaxis in the UK, 1992-2001. Novartis Found Symp 2004, 257:116-128.

20. Helbling A, Hurni T, Mueller UR, Pichler WJ: Incidence of anaphylaxis with circulatory symptoms: a study over a 3-year period comprising 940,000 inhabitants of the Swiss Canton Bern. Clin Exp Allergy 2004, 34:285-290.

21. Poulos LM, Waters A-M, Correl PK, et al.: Trends in hospitalizations for anaphylaxis, angiooedema, and urticaria in Australia, 1993-1994 to 2004-2005. J Allergy Clin Immunol 2007, 120:878-884.

22. Gupta R, Sheikh A, Strachan DP, Anderson HR: Time trends in allergic disorders in the UK. Thorax 2007, 62:91-96.

23. Lin RY, Shah SN: Increasing hospitalizations due to angioedema in the United States. Ann Allergy Asthma Immunol 2008, 101:185-192.

24. Lin RY, Anderson AS, Shah SN, Nurruzzaman F: Increasing anaphylaxis hospitalizations in the first 2 decades of life: New York State, 1990-2006. Ann Allergy Asthma Immunol 2008, 101:387-393.

25. US Census Bureau: Population estimates. Available at http://www. census.gov/popest/states/asrh/files/SC-EST2009-AGESEX-RES.csv. Accessed July 30, 2010.
26. US Census Bureau: Population estimates for the U.S., regions, divisions, and states by 5 -year age groups and sex: Time series estimates, July 1, 1990 to July 1, 1999 and April 1, 1990 Census population counts. Available at http://www.census.gov/popest/ archives/1990s/ST-99-08.txt. Accessed July 30, 2010.

27. Florida Population Estimates, Florida CHARTS (Community Health Assessment Resource Tool Set), Florida Department of Health, Office of Health Statistics \& Assessment. Available at http://www.floridacharts.com/charts/PopQuery.aspx and at http:// hpe04sdbsdev/FLCHARTS/Population/PopulationRpt.aspx. Accessed September 29, 2010.

28. Camargo CA Jr, Clark S, Kaplan MS, et al.: Regional differences in EpiPen prescriptions in the United States: the potential role of vitamin D. J Allergy Clin Immunol 2007, 120:131-136.

29. Mullins RJ, Clark S, Camargo CA Jr: Regional variation in epinephrine autoinjector prescriptions in Australia: more evidence for the vitamin D-anaphylaxis hypothesis. Ann Allergy Asthma Immunol 2009, 103:488-495.

30. Rudders SA, Espinola JA, Camargo CA Jr: North-south differences in US emergency department visits for acute allergic reactions. Ann Allergy Asthma Immunol 2010, 104:413-416.

31. Allison PD: Poisson regression. In Logistic Regression Using the SAS System: Theory and Application. Cary, NC: SAS Institute; 1999:217-231.

32. Szklo M, Nieto FJ: Epidemiology Beyond the Basics. Gaithersburg: Aspen; 2000:310-311.

33. Greenland S: Modeling and variable selection in epidemiologic analysis. Am J Public Health 1989, 79:340-349.

34. Fisher ES, Whaley FS, Krushat WM, et al.: The accuracy of Medicare's hospital claims data: progress has been made, but problems remain. Am J Public Health 1992, 82:243-248.

35. Lloyd SS, Rissing JP: Physician and coding errors in patient records. JAMA 1985, 254:1330-1336.

36. Institute of Medicine, National Academy of Sciences. Reliability of Medicare hospital discharge records: report of a study 1977. Available at http://www.nap.edu/catalog.php?record id=9930\#toc. Accessed July 21, 2010.

37. Lydon-Rochelle MT, Holt VL, Nelson JC, et al.: Accuracy of reporting maternal in-hospital diagnoses and intrapartum procedures in Washington state linked birth records. Paediatr Perinat Epidemiol 2005, 19:460-471.

38. Yasmeen S, Romano PS, Schembri ME, et al.: Accuracy of obstetric diagnoses and procedures in hospital discharge data. Am J Obstet Gynecol 2006, 194:992-1001.

39. Kelsey JL, Whittemore AS, Evans AS, Thompson WD: Methods in Observational Epidemiology, edn 2. New York: Oxford University Press; 1996:342-348.

40. West SL, D'Aloisio AA, Ringel-Kulka T, et al.: Population based drug-related anaphylaxis in children and adolescents captured by South Carolina Emergency Room Hospital Discharge Database (SCERHDD) (2000-2002). Pharmacoepidemiol Drug Saf 2007, 16:1255-1267. 